\title{
The gendered nature and malleability of gamer stereotypes
}

\author{
Thekla Morgenroth (corresponding author) \\ University of Exeter \\ Washington Singer Laboratories, University of Exeter, Perry Road, Exeter, EX4 4QG, \\ UK. E-mail: T.Morgenroth@exeter.ac.uk; Phone: +44-7453-585835
}

\author{
Michelle Stratemeyer \\ University of Melbourne, Centre for Ethical Leadership \\ Benjamin Paaßen \\ University of Bielefeld
}

\begin{abstract}
Author Note:
The first author of this paper uses they/them/their pronouns, the second author uses she/her/hers pronouns, and the third author uses he/him/his pronouns.
\end{abstract}




\begin{abstract}
Video gaming is seen as a male space. Female gamers are seen as atypical, have their competence challenged, and face more harassment than male gamers do. This precarious position is increasingly problematic as video gaming is now one of the most prevalent leisure activities, providing an opportunity to both forge and maintain friendships, and to achieve social status and career opportunities. We argue that the marginalization of female gamers is driven by masculine gamer stereotypes. We investigate the content and gendered nature of gamer stereotypes as well as their malleability in response to exposure to female gamers across two studies $\left(N_{\text {Study }}=287\right.$; $\left.N_{\text {Study } 2}=176\right)$. We explore the content of gamer stereotypes and find that they contain both negative aspects, such as lacking social skills, and positive aspects, such as being competent and agentic. Both studies demonstrate that gamer stereotypes are more similar to stereotypes of men and boys than those of women and girls. In Study 2 we test whether exposure to a female gamer can change the negative association between female stereotypes and gamer stereotypes, finding support for this prediction. We conclude that gamer stereotypes are highly gendered but may be malleable: increasing the visibility of female gamers could potentially reduce the incompatibility between femininity and gaming.
\end{abstract}

Keywords: Gaming, gamer, gender, stereotypes, video games 
Despite the fact that the percentage of female gamers has increased over the years ${ }^{1}$, women continue to face harassment and are challenged over their legitimacy and competence in gaming spaces ${ }^{2,3}$. In turn, they disengage from the gaming community, resulting in fewer benefits such as meaningful social ties ${ }^{4}$.

One of the factors affecting these dynamics may be gamer stereotypes. Gamers are generally perceived to be mostly young, isolated $\mathrm{men}^{5}$, who are unpopular and introverted ${ }^{6}$. These stereotypes are in direct opposition to stereotypes of women, who are perceived as communal and socially skilled ${ }^{7}$.

The stereotype itself is somewhat surprising, since almost half of the people who play video games are female ${ }^{8}$. One of the reasons for this may be that stereotypes lag behind any cultural changes ${ }^{9}$. Moreover, Paaßen and colleagues ${ }^{8}$ suggest that the masculine gamer stereotype is driven by the fact that almost all highly visible gamers - such as gamers streaming on twitch - are male. In other words, the gamer stereotype is misrepresenting the gaming community because female gamers are less visible. By extension, if people are exposed to more female gamers, gamer stereotypes may change.

Our objectives are to explore the content and gendered nature of gamer stereotypes, and test whether female representation can shift this perception. We build on previous research on gamer stereotypes ${ }^{6}$ by using a wider range of attributes and examine stereotypes of teenagers as well as adults because many adolescents engage in gaming ${ }^{10}$ and because many gamers start gaming during their teenage years.

We predict the following:

H1: Gamer stereotypes will be more compatible with male stereotypes than with female stereotypes. 
H2: Exposure to a female gamer (vs. a male gamer or no gamer) will change gamer stereotypes to become more compatible with female stereotypes.

The data sets used in this paper are available at https://osf.io/jfh9b/?view_only=f7de84580b8242e5b965a3b32ecdb6b9. All materials can be found in the online supplement.

\section{Study 1}

We pre-registered this study ${ }^{\mathrm{a}}$ (see https://osf.io/nwh89/) and tested H1 ${ }^{\mathrm{b}}$.

\section{Method}

Participants. We recruited participants from Western, English-speaking countries via the Prolific website. Our final sample consisted of $207^{\mathrm{c}}$ participants $\left(55 \%\right.$ women; $M_{\text {Age }}=36.48$, $S D=11.93)$, predominantly from the UK $(71 \%)$ and the US $(21 \%) ; 36 \%$ of our sample identified as gamers. $^{\mathrm{d}}$

Design and materials. Participants were randomly assigned to one of five conditions ${ }^{\mathrm{e}}$, rating how typical a range of attributes were of one out of five groups: gamers, women, men,

${ }^{a}$ Please note that only Study 1 was pre-registered, as Study 2 was conducted before Study 1 . We changed the order in which we describe the studies for narrative purposes.

${ }^{\mathrm{b}}$ We also investigated whether male gamers, compared to female gamers, were seen as more similar to gamers. Results can be found in the online supplement.

${ }^{\mathrm{c}}$ We collected data from 80 additional participants for two additional conditions to investigate the perception of female and male gamers. Results can be found in the online supplement

${ }^{\mathrm{d}}$ Exploratory analyses split by gamer status and gender can be found in the online supplement.

e As our pre-registration states, we had originally planned to combine the collected data with those from the prestudy of Study 2 (see below). However, as we judged the samples to be too different from each other, we collected data for all conditions and did not use data from the pre-study. 
teenage girls, or teenage boys. For the gamer condition, we included a definition of gamer:

"When thinking of gamers, think of someone who identifies as a gamer and who regularly plays video games on a video game console (e.g., Playstation, Xbox, Nintendo) or on a PC.”

Participants were presented with the Schein Descriptive Index ${ }^{11}$ containing 92 attributes (e.g., "analytical"), an additional 16 attributes that had been identified as being part of gamer stereotypes $^{6}$ (e.g., "obsessive") and two attention checks items ("alive", "human") in random order. For each attribute, participants indicated how characteristic it was of the group they were assigned, on a scale from 1 (not characteristic) to 5 (characteristic).

\section{Results}

Before testing our hypotheses, we explored the content of gamer stereotypes. Following the method used by Ryan and colleagues ${ }^{12}$, we performed a series of one-sample $t$-tests to identify attributes that were seen as stereotypical (i.e., significantly above the midpoint) or counter-stereotypical (i.e., significantly below the midpoint) of gamers (see Table 1).

Many attributes (e.g., reclusive, isolated) indicate a lack of communality ${ }^{7,13}$. Moreover, many of the stereotypical attributes were related to facets of masculinity such as competence ${ }^{13}$ (e.g., intelligent, analytical ability) or agency $^{7}$ (e.g., assertive, competitive), suggesting that gamer stereotypes are indeed masculine.

To test H1, we re-structured our data file such that each attribute rather than each participant was now one case (i.e., row), while the mean rating of these attributes for each group (i.e., gamers, men, women, teenage boys, teenage girls) were the different variables (i.e., columns) ${ }^{11}$. We used this dataset to run two multiple linear regressions (one with ratings of women and men as predictors of gamer attributes; one with ratings of teenage girls and teenage 
boys as predictors). We calculated $95 \%$ confidence intervals to assess whether the two coefficients were statistically different from each other ${ }^{14}$.

In line with $\mathrm{H} 1$, there was a positive association of what was seen as typical of men and gamers, while the association was negative for the ratings of women and gamers. Similarly, we found a positive association of what was seen as typical of teenage boys and gamers, while the association was negative for the ratings of teenage girls and gamers (see Table 2). In both cases, the coefficients were different from each other.

\section{Discussion}

We found that gamer stereotypes are indeed gendered: while stereotypes of men and teenage boys are compatible with stereotypes of gamers, stereotypes of women and teenage girls are incompatible with stereotypes of gamers. Interestingly, while gamer stereotypes included a range of negative attributes that were previously identified in the literature ${ }^{6}$, they also included positive characteristics such as agency and competence.

\section{Study 2}

In this study, we seek to replicate the findings regarding the gendered nature of gamer stereotypes and investigate whether exposure to a female gamer can change these stereotypes.

\section{Method}

Participants. 244 psychology students from a UK university participated in this study as part of a class. We excluded 67 participants for failing the attention check or not following instructions. Our final sample consisted of 176 participants (84\% women; 74\% British; 9\% gamers; $\left.M_{\text {Age }}=19.02 ; S D=2.67\right)$.

Design and procedure. Data were collected as part of one of the participants' classes. Three groups (all enrolled in the same class) participated on two consecutive days with each 
class being assigned to one condition. Students were randomly allocated to groups and told that we were interested in how different videos could affect the perception of groups. Participants were shown one of three 10-minute videos. In the two experimental conditions, this was a video featuring either a male or a female actor playing a sequence of the game Mass Effect 2. This game was selected to cover multiple 'core game' genres ${ }^{15}$ (shooter, action, and roleplaying games). The video showed the game play footage with an overlay of the actor's face (videos are available here: https:/www.youtube.com/watch?v=rlRfLy6P25g and here:

https://www.youtube.com/watch?v=UdokRUVnld4 ). The gaming footage was identical, as were the comments the two actors made. Pilot testing $(N=41)$ showed that the two videos were seen as equally authentic, easy to follow, and entertaining. The gamers were seen as equally competent. The only difference was that the female gamer was rated as marginally more likable (5.10 vs. 4.20 on a seven-point scale). In the control condition, participants watched video unrelated to gaming: a documentary about animals.

Participants then rated what gamers were like using the same attributes as in Study 1. Moreover, participants in the two experimental conditions were asked whether they thought the person in the video they watched was a gamer.

\section{Results}

Stereotypes of gamers were fairly consistent across the two studies $r(108)=.86, p<.001$ (see Table 3).

We combined the data set with the data set from a pre-study with a similar sample (179 UK students; $51 \%$ women, $M_{A g e}=24.88 ; S D=7.04$ ), in which we asked participants to rate how typical the attributes were of women, men, teenage girls, and teenage boys using the same methodology as Study 1. 
To test H1, we first tested whether there were differences in whether or not the female and male actors were seen as gamers. Despite the videos being almost identical, 41 of 62 participants indicated that they considered the female actress a gamer, whereas 52 of 59 participants considered the male actor a gamer, $\chi^{2}(1)=8.23 ; p=.005$, supporting H1.

Next, we tested H1 using the same analytic strategy as in Study 1 using gamer ratings from the control condition (see Table 4). There was a negative association of what was seen as typical of women and gamers. However, while the association was positive for the ratings of men and gamers, it was not significant. As the confidence intervals overlapped by less than 50\%, the coefficients can nevertheless be considered different from each other ${ }^{14}$, supporting H1.

Results for teenager stereotypes were also in line with $\mathrm{H} 1$. We found a positive association of what was seen as typical of teenage boys and gamers, while the association was negative for the ratings of teenage girls and gamers.

To test whether exposure to a female gamer would eliminate the incompatibility of gamer stereotypes and female stereotypes we ran the same regressions described above but with ratings of gamers made by participants who had watched the female gamer video (see Table 4, also for results regarding the male gamer condition). ${ }^{\mathrm{f}}$ In line with $\mathrm{H} 2$, the negative association of what was seen as typical of women and gamers was no longer significant. However, we found a positive association between the ratings of men and gamers, a surprising finding given that this association was not significant in the control condition. Similarly, while the negative association between ratings of teenage girls and gamers was now only marginally significant, the difference

\footnotetext{
${ }^{\mathrm{f}}$ While an ANOVA might seem more intuitive to test whether there are differences between groups, the structure of our data (with each attribute, rather than each participant, being one case) makes this analysis impossible.
} 
between coefficients remained significant, as the association between ratings of teenage boys and gamers was positive and significant.

\section{Discussion}

We found that gamer stereotypes are more compatible with male gender stereotypes than with female gender stereotypes, replicated the finding from Study 1 in a different sample. Gamer stereotypes were generally consistent across the two samples, indicating that there is widespread agreement about what gamers are like.

The evidence regarding the beneficial effects of exposure to female gamers was mixed. The incompatibility of female stereotypes and gamer stereotypes disappeared after exposure to a female gamer. However, exposure to a female gamer also increased the stereotype compatibility of men and gamers. As this also occurred in the male gamer condition, it may be due to the association of gaming content featured in the video (e.g., violence) with masculinity ${ }^{16}$. Future research should investigate how gamer gender and the masculinity/femininity of the gaming content and game genre interact and inform gamer stereotypes.

\section{General Discussion}

Across two studies, we found that gamer stereotypes are gendered, but also malleable. However, our studies have a number of limitations. We only considered short-term exposure to a female gamer. Given the promising effects demonstrated, it may be that longer exposure may have stronger effects. Moreover, we focused on gamer stereotypes in a Western, English-speaking context. Future research should investigate whether these stereotypes generalize across cultures.

Despite these limitations, our findings have important implications. Masculine stereotypes of different roles have been shown to keep women out of domains such as 
leadership ${ }^{17}$ and STEM fields ${ }^{18}$. It is likely that similar processes apply to gaming spaces. This is problematic given the benefits associated with gaming among men such as social connections and belonging ${ }^{3}$. Additionally, gaming is developing into its own stand-alone career, and may be associated with increasing interest in, and relevant skills for, STEM occupations ${ }^{19,20}$. Our research demonstrates that adequate representation of female gamers could alleviate this problem and make gaming more inclusive. 


\section{References}

1. Statista. Distribution of computer and video gamers in the United States from 2006 to 2019, by gender 2020; Retrieved from https:/www.statista.com/statistics/232383/gendersplit-of-us-computer-and-video-gamers/

2. Consalvo M. Confronting toxic gamer culture: A challenge for feminist game scholar. Ada: A Journal of Gender, New Media, and Technology 2012; 1. doi:10.7264/N33X84KH

3. Kuznekoff J, Rose L. Communication in multiplayer gaming: Examining player responses to gender cues. New Media \& Society 2012; 15(4):541-56. doi: $10.1177 / 1461444812458271$

4. Lenhart A, Smith A, Anderson M, Duggan M, Perrin A. (2015) Teens, technology, and friendships. Washington, DC, USA: Pew Research Center. Retrieved from: http://www.pewinternet.org/2015/08/06/teens-technology-and-friendships/

5. Williams D, Yee N, Caplan SE. Who plays, how much, and why? Debunking the stereotypical gamer profile. Journal of Computer-Mediated Communication 2008; 13(4): 993-1018. doi:10.1111/j.1083-6101.2008.00428.x

6. Kowert R, Griffiths, M, Oldmeadow, JA. Geek or chic? Emerging stereotypes of online gamers. Bulletin of Science, Technology \& Society 2012; 32(6):471-9. doi:10.1177/0270467612469078

7. Eagly, AH. (1987) Sex differences in social behavior: A social-role interpretation. Hillsdale, NJ, USA: Erlbaum. 
8. Paaßen B, Morgenroth T, Stratemeyer M. What is a true gamer? The male gamer stereotype and the marginalization of women in video game culture. Sex Roles 2017; 76(7-8): 421-35. doi:10.1007/s11199-016-0678-y

9. Diekman AB, Eagly AH, Johnston AM. Social structure. In: Dovidio JF, Hewstone M, Glick PG, \& Esses VM, eds. The Sage handbook of prejudice, stereotyping, and discrimination. Thousand Oaks, CA: Sage, pp 209-224.

10. Anderson M, Jiang, J. (2018). Teens, Social media \& Technology. Washington, DC, USA: Pew Research Center. Retrieved from: https://www.pewinternet.org/2018/05/31/teens-social-media-technology-2018/

11. Schein V. The relationship between sex role stereotypes and requisite management characteristics. Journal of Applied Psychology 1973; 57(2): 95-100. doi:10.1037/h0037128

12. Ryan MK, Haslam SA, Hersby MD, Bongiorno R. Think crisis-think female: The glass cliff and contextual variation in the think manager-think male stereotype. Journal of Applied Psychology 2011; 96(3):470-484.

13. Fiske S, Cuddy AJC, Glick P. Universal dimensions of social cognition: warmth and competence. Trends in Cognitive Sciences 2007; 11(2): 77-83. doi:10.1016/j.tics.2006.11.005

14. Cumming G. Inference by eye: reading the overlap of independent confidence intervals. Statistics in medicine 2008; 28(2): 205-220. doi:10.1002/sim.3471

15. Vermeulen L, Van Looy J, De Grove F, Courtois C. (2011). You are what you play? A quantitative study into game design preferences across gender and their interaction with gaming habits. In: Copier M, Waern A, Kennedy H, eds. Proceedings of the 2011 DiGRA 
International Conference: Think Design Play. Utrecht, Netherlands: Digital Games Research Association (DiGRA), pp. 1-20.

16. Mahalik JR, Locke BD, Ludlow LH, et al. Development of the Conformity to Masculine Norms Inventory. Psychology of Men \& Masculinity 2003; 4(1): 3-25.doi:10.1037/15249220.4.1.3

17. Eagly AH, Karau SJ. Role congruity theory of prejudice towards female leaders. Psychological Review 2002; 109(3): 573-598.

18. Cheryan S, Master A, Meltzoff AN. Cultural stereotypes as gatekeepers: Increasing girls' interest in computer science and engineering by diversifying stereotypes. Frontiers in Psychology 2015; 6(49). doi:10.3389/fpsyg.2015.00049

19. Giammarco E, Schneider T, Carswell J, Knipe W. Video game preferences and their relation to career interests. Personality and Individual Differences 2015; 73: 98-104. doi:10.1016/j.paid.2014.09.036

20. Mayo MJ. Video games: A route to large-scale STEM education? Science 2009; 323(5910): 79-82. doi:10.1126/science.1166900 